\title{
Address Assignment and Routing Schemes for ZigBee-Based Long-Thin Wireless Sensor Networks
}

\author{
Meng-Shiuan Pan*1, Hua-Wei Fang ${ }^{\dagger \dagger 2}$, Yung-Chih Liu ${ }^{\dagger 3}$, and Yu-Chee Tseng*4 \\ ${ }^{*}$ Department of Computer Science \\ National Chiao Tung University, Hsin-Chu, Taiwan \\ $\dagger$ Networks and Multimedia Institute \\ Institute for Information Industry, Taipei, Taiwan \\ $\ddagger$ Department of Computer Science and Information Engineering \\ National Taiwan University, Taipei, Taiwan \\ Email: $\left\{{ }^{1}\right.$ mspan, ${ }^{4}$ yctseng $\} @$ cs.nctu.edu.tw, ${ }^{2}$ d95030@ @ csie.ntu.edu.tw, ${ }^{3}$ ulysses@nmi.iii.org.tw
}

\begin{abstract}
Wireless sensor networks (WSNs) have been extensively researched recently. This paper makes two contributions to this field. First, we promote a new concept of long-thin (LT) topology for WSNs, where a network may have a number of linear paths of nodes as backbones connecting to each other. These backbones are to extend the network to the intended coverage areas. At the first glance, a LT WSN only seems to be a special case of numerous WSN topologies. However, we observe, from real deployment experiments, that such a topology is quite general in many applications and deployments. The second contribution is that we show that the address assignment and thus the tree routing scheme defined in the original ZigBee specification may work poorly, if not fail, in a LT topology. We thus propose simple, yet efficient, address assignment and routing schemes for a LT WSN. Simulation results and prototyping experiences are also reported.
\end{abstract}

Index Terms-address assignment, long-thin network, routing protocol, wireless sensor network, ZigBee.

\section{INTRODUCTION}

The rapid progress of wireless communication and embedded micro-sensing MEMS technologies has made wireless sensor networks (WSN) possible. A WSN usually needs to configure itself automatically and support ad hoc routing. A lot of research works have been dedicated to WSNs, including power management [15], routing and transportation [3], coverage issue [7], and localization [1]. In the application side, habitat monitoring is explored in [4], wildfire monitoring is addressed in [6], and navigation is studied in [14].

Recently, several WSN platforms have been developed, such as MICA [11] and Dust Network [5]. For interoperability among different systems, standards such as ZigBee [17] have been developed. In the ZigBee protocol stack, physical and MAC layer protocols are adopted from the IEEE 802.15.4 standard [8]. ZigBee solves interoperability issues from the physical layer to the application layer.

ZigBee supports three kinds of networks, namely star, tree, and mesh networks. A ZigBee coordinator is responsible for initializing, maintaining, and controlling the network. A star network has a coordinator with devices directly connecting to the coordinator. For tree and mesh networks, devices can communicate with each other in a multihop fashion. The network is formed by one ZigBee coordinator and multiple ZigBee routers. A device can join a network as an end device by associating with the coordinator or a router. In ZigBee, a device is said to join a network successfully if it can obtain a 16-bit network address from the coordinator or a router. ZigBee specifies a distributed address assignment scheme, which allows a parent device to locally compute addresses for child devices. While the assignment scheme has low complexity, it also prohibits the network from scaling up and thus cannot be used in LT networks.

In this paper, we discuss the LT network, which is considered as a specific but common network topology in many surveillance applications, such as gas leakage detection of fuel pipes, carbon dioxide concentration monitoring in tunnels, stage measurements in sewers, street lights monitoring in highway systems, flood protection of rivers, and vibration detection of bridges. In such a network, nodes may form several long backbones and these backbones are to extend the network to the intended coverage areas. A backbone is a linear path which may contain tens or hundreds of $\mathrm{ZigBee}$ routers. So the network can be scaled up with limited hardware cost.

In this work, we propose address assignment and routing schemes for ZigBee-based LT WSNs. To assign addresses to nodes, we design rules to divide nodes into clusters. Each node belongs to one cluster and each cluster has a unique cluster $I D$. All nodes in a cluster will have the same cluster ID, but different node IDs. The structure of ZigBee network address is divided into two parts: one is cluster $I D$ and the other is node ID. Following the same ZigBee design philosophy, the proposed scheme is simple and has low complexity. Existing works [2][12][13][16] have discussed address assignment for WSNs, but they are not designed for ZigBee or LT WSNs. To the best of our knowledge, this is the first work addressing this issue. Moreover, similar to the ZigBee tree routing protocol, the proposed routing protocol can also utilize nodes' network addresses to facilitate routing. In addition, routing can take advantage of shortcuts for better efficiency, so our scheme does not restrict nodes to relay packets only to their parent or child nodes as ZigBee does.

The rest of this paper is organized as follows. Preliminaries 
are given in Section II. Section III presents our algorithms. Section IV presents some performance evaluations. Finally, Section $\mathrm{V}$ concludes this paper.

\section{PRELIMINARIES}

\section{A. ZigBee Address Assignment}

In ZigBee, network addresses are assigned to devices by a distributed address assignment scheme. Before forming a network, the coordinator determines the maximum number of children of a router $(\mathrm{Cm})$, the maximum number of child routers of a router $(R m)$, and the depth of the network $(\mathrm{Lm})$. Note that a child of a router can be a router or an end device, so $C m \geq R m$. The coordinator and routers can each have at most $R m$ child routers and at least $C m-R m$ child end devices. Devices' addresses are assigned in a top-down manner. For the coordinator, the whole address space is logically partitioned into $R m+1$ blocks. The first $R m$ blocks are to be assigned to the coordinator's child routers and the last block is reserved for the coordinator's own child end devices. From $\mathrm{Cm}, \mathrm{Rm}$, and $L m$, each router computes a parameter called Cskip to derive the starting addresses of its children's address pools. The Cskip for the coordinator or a router in depth $d$ is defined as:

$$
\operatorname{Cskip}(d)=\left\{\begin{array}{lc}
1+C m \times(L m-d-1) & \text { if } R m=1 \\
\frac{1+C m-R m-C m R m^{L m-d-1}}{1-R m} & \text { otherwise. }
\end{array}\right.
$$

The coordinator is said to be at depth $d=0$, and $d$ is increased by one after each level. Address assignment begins from the ZigBee coordinator by assigning address 0 to itself. If a parent node at depth $d$ has an address $A_{\text {parent }}$, the $n$-th child router is assigned to address $A_{\text {parent }}+(n-1) \times \operatorname{Cskip}(d)+1$ and $n$-th child end device is assigned to address $A_{\text {parent }}+$ $R m \times C \operatorname{skip}(d)+n$. An example of the address assignment is shown in Fig. 1. The Cskip of the coordinator is obtained from Eq. (1) by setting $d=0, C m=5, R m=4$, and $L m=2$. Then the child routers of the coordinator will be assigned to addresses $0+(1-1) \times 6+1=1,0+(2-1) \times 6+1=7$, $0+(3-1) \times 6+1=13$, and etc. The address of the only child end device of the coordinator is $0+4 \times 6+1=25$. Note that the length of a network address is 16 bits; thus, the maximum address capacity is $2^{16}=65536$. Obviously, the above assignment is much suitable for regular networks, but not for LT WSNs. For example, when setting $C m=R m=2$, the depth of the network can only be 15 . Also, when there is a LT backbone, the address space is not well utilized.

\section{B. ZigBee Tree Routing Protocol}

In a ZigBee network, the coordinator and routers can directly transmit packets along the tree without using any route discovery. When a device receives a packet, it first checks if it is the destination or one of its child end devices is the destination. If so, this device will accept the packet or forward this packet to the designated child. Otherwise, it forwards the packet to its parent. Assume that the depth of this device is $d$ and its address is $A$. This packet is for one

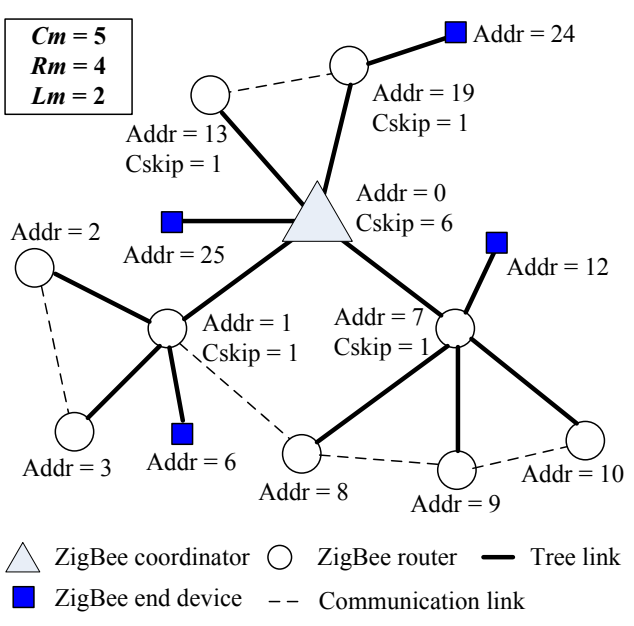

Fig. 1. A ZigBee address assignment example.

of its descendants if the destination address $A_{\text {dest }}$ satisfies $A<A_{\text {dest }}<A+C \operatorname{skip}(d-1)$, and this packet will be relayed to the child router with address

$$
A_{r}=A+1+\left\lfloor\frac{A_{\text {dest }}-(A+1)}{C \operatorname{skip}(d)}\right\rfloor \times C \operatorname{skip}(d) .
$$

If the destination is not a descendant of this device, this packet will be forwarded to its parent. In the ZigBee tree routing, each node can only choose its parent or child as the next node. This strategy may cause long delay in LT networks.

\section{THE PRoposed SCHEMES}

Assuming that all nodes are router-capable devices, we show how to form a LT WSN (as in Fig. 2(a)). Nodes are divided into multiple clusters, each as a line segment. For each cluster, we define two special nodes, named cluster head and bridge. The cluster head (resp., the bridge) is the node that has the smallest (resp., largest) hop count to the coordinator. As a special case, the coordinator, is also considered as a cluster head. The other nodes are network nodes (refer to Fig. 2(b)). A cluster $C$ is a child cluster of a cluster $C^{\prime}$ if the cluster head of $C$ is connected to the bridge of $C^{\prime}$. Reversely, $C^{\prime}$ is $C^{\prime}$ s parent cluster. Note that a cluster must have a linear path as its subgraph. But it may have other extra links beside the linear path. For example, in Fig. 2(b), there are two extra radio links $(A, A 2)$ and $(A 1, A 3)$ in $A$ 's cluster. To be compliant with ZigBee, we divide the ZigBee 16-bit network address into two parts, an $m$-bit cluster ID and a $(16-m)$-bit node ID. The network address of a node $v$ is thus expressed as $\left(C_{v}, N_{v}\right)$, where $C_{v}$ and $N_{v}$ are $v$ 's cluster ID and node ID, respectively.

\section{A. Network Planning}

Before deploying a network, the network manager should carefully plan the placement of cluster head, bridge, and network nodes. There are some basic principles:

1) The network contains a number of linear paths.

2) For each cluster, the first and the last nodes are preassigned (manually) as cluster head and bridge, respectively. 


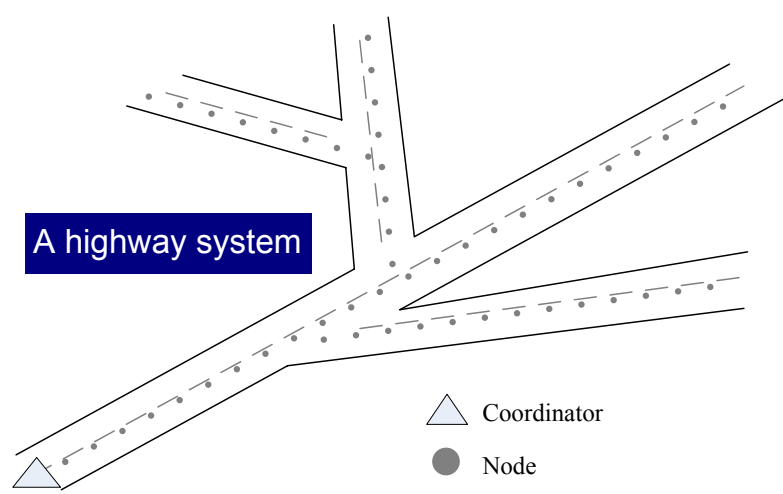

(a)

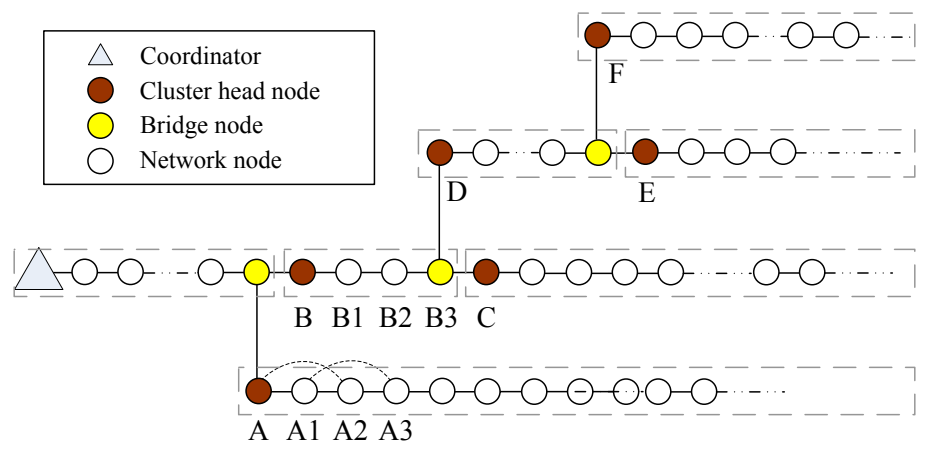

(b)

Fig. 2. (a) A LT WSN. (b) Role assignment.

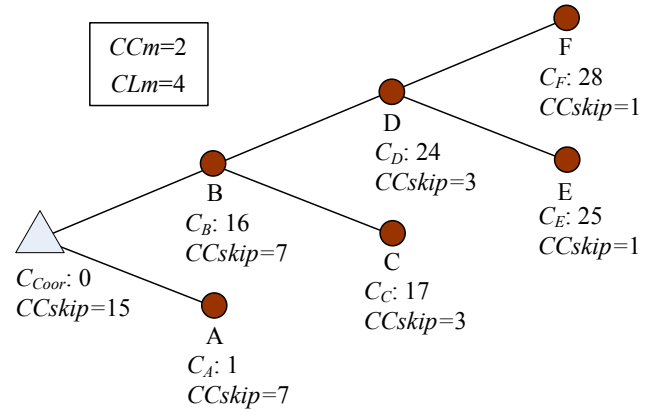

Fig. 3. The logical network of Fig. 2(b).

3) A cluster head that is not the coordinator should have a link to the bridge of its parent cluster.

4) Conversely, the bridge of a cluster which has child clusters should have a link to the cluster head of each child cluster.

5) A cluster does not cross other clusters and does not have links with other clusters except those locations nearby the cluster head and bridge areas.

After planing the placements, the network manager can construct a logical network $G_{L}$, in which each cluster is converted into a single node and the parent-child relationships of clusters are converted into edges. For example, Fig. 3 is the logical network of Fig. 2(b). Then the network manager can determine two parameters: the maximum number of children $C \mathrm{Cm}$ of a node in $G_{L}$ and the depth $C L m$ of $G_{L}$.

\section{B. Address Assignment}

Network addresses are assigned in two stages. With the above planning, the network manager first mutually assigns a cluster ID to each node. Then node IDs are assigned in a distributed manner after network deployment.

The network manager assigns cluster IDs as follows. If $C C m=1$, there is no branch in the network and the cluster ID assignment is a trivial case. If $C C m \geq 2$, the cluster IDs are assigned following the style of ZigBee in a recursive manner. The nodes in the coordinator's cluster have a cluster
ID of 0 . For each node at depth $d$ in $G_{L}$, if its cluster ID is $C$, then its $i$-th child cluster is assigned a cluster ID of $C+(i-1) \times C C \operatorname{skip}(d)+1$, where

$$
C C \operatorname{skip}(d)=\frac{1-C C m^{C L m-d}}{1-C C m} .
$$

Fig. 3 shows an example of cluster ID assignment of Fig. 2(b). Note that, in our scheme, for a cluster head in $G_{L}$ with depth $d$, we also set each node in this cluster has a logical depth $d$.

After cluster ID assignment and node deployment, each node periodically broadcasts HELLO packets including its IEEE 64-bit MAC address, 16-bit network address (with its cluster ID but node ID is initiated to NULL), and role. Each node maintains a neighbor table to record its neighbors' information. Then the node ID assignment is started by the coordinator broadcasting its beacon with its node ID setting to 0 . When a node without a node ID receives a beacon, it will send an Association_Request to the beacon sender. If there are multiple beacons, the node with the strongest signal strength will be selected. When the beacon sender, say, $v$ with a network address $\left(C_{v}, N_{v}\right)$, receives the association request(s), it will do the following steps.

1) If $v$ is not a bridge node, it sets a parameter $N=N_{v}+1$. Then it sorts request senders according to the received signal strength of their request packets in an descending order into a list $L$. Then $v$ sequentially examines each node $u \in L$ by the following rules:

- If $C_{u} \neq C_{v}, v$ skips $u$ and continues to examine the next node in $L$.

- Otherwise, $v$ assigns $N_{u}=N$ and increments $N$ by 1 . Then $v$ replies an Association_Response to $u$ with its address. If $L$ is not empty, $v$ loops back and continues to examine the next node in $L$.

After finishing the above iteration, $v$ further selects a node $u$, from the accepted ones, using the following rules: i) If there is a bridge node in the accepted ones, $v$ selects the bridge node. ii) Otherwise, $v$ selects the last node in $L$. Then $v$ delegates $u$ as the next beacon sender by sending a command next_beacon_sender $(u)$ to $u$. 
TABLE I

PART OF THE RESUlTING NETWORK ADDRESSES IN FIG. 2.

\begin{tabular}{|c|c|c|c|c|c|}
\hline \multirow{2}{*}{ Node } & \multicolumn{2}{|c|}{ Network address } & \multirow{2}{*}{ Node } & \multicolumn{2}{c|}{ Network address } \\
\cline { 2 - 4 } & Cluster ID & Node ID & & Cluster ID & Node ID \\
\hline \hline A & 00001 & 00000000000 & B & 10000 & 00000000000 \\
\hline A1 & 00001 & 00000000001 & B1 & 10000 & 00000000001 \\
\hline A2 & 00001 & 00000000010 & B2 & 10000 & 00000000010 \\
\hline A3 & 00001 & 00000000011 & B3 & 10000 & 00000000011 \\
\hline
\end{tabular}

2) If $v$ is a bridge node, it only accepts the requests from cluster heads of its child cluster. When deciding to accept a node $u, v$ replies an Association_Response to $u$ with $N_{u}=0$ and a next_beacon_sender $(u)$.

For each node $u$ that receives a next_beacon_sender $(u)$ in the above steps, it will use the MLME-START primitive defined in IEEE 802.15.4 to start its beacons. Then the same procedure is repeated. Note that we allow a beacon sender to accept multiple children so as to reduce the communication cost of address assignment. Table I shows parts of the address assignment results in Fig. 2(b).

\section{Routing Rules}

Routing in our LT WSN can be purely based on the above address assignment results. Through HELLO packets, a node can collect its neighbors' network addresses. Suppose that a node $v$ at logical depth $d$ receives a packet with a destination address $\left(C_{\text {dest }}, N_{\text {dest }}\right)$. If $v$ is the destination, it simply accepts this packet. Otherwise, $v$ performs the following procedures.

1) If the destination is a neighbor of $v, v$ sends this packet to the destination directly.

2) If $C_{d e s t}=C_{v}$, the destination is within the same cluster. Node $v$ can find an ancestor or a descendant in its neighbor table, say, $u$ such that $C_{u}=C_{d e s t}$ and the value of $\left|N_{u}-N_{\text {dest }}\right|$ is minimized, and forward this packet to $u$.

3) If $C_{\text {dest }}$ is a descendant cluster of $C_{v}$, i.e., $C_{v}<C_{\text {dest }} \leq$ $C_{v}+(C C m-1) \times C C \operatorname{skip}(d)+1$, then $v$ checks if it has a neighbor $u$ which satisfies $C_{u} \leq C_{\text {dest }} \leq C_{u}+$ $(C C m-1) \times C C \operatorname{skip}(d+1)+1$. If such a $u$ exists, then $v$ forwards the packet to $u$. In case that there are multiple candidates, the one with the smallest $\mid N_{u}-$ $N_{\text {dest }} \mid$ is selected. Otherwise, $v$ finds a neighbor $u$ which is located in the same cluster and has the maximum $N_{u}$ and forwards the packet to $u$.

4) For all other cases, $C_{\text {dest }}$ must be an ancestor cluster of $C_{v}$ or not within the same logical subtree. Then $v$ checks if it has a neighbor $u$ which satisfies $C_{u}<C_{v} \leq$ $C_{u}+(C C m-1) \times C C \operatorname{skip}(d-1)+1$. If such a $u$ exists, $v$ forwards the packet to $u$. Note that the above condition confines that $C_{u}$ is the parent cluster of $C_{v}$. Otherwise, $v$ finds a neighbor $u$ which is located in the same cluster and has the minimum $N_{u}$ and forwards the packet to $u$.

Note that the above design tries to make a balance between efficiency and simplicity. It basically follows the ZigBee treelike routing. However, making shortcut along the linear paths

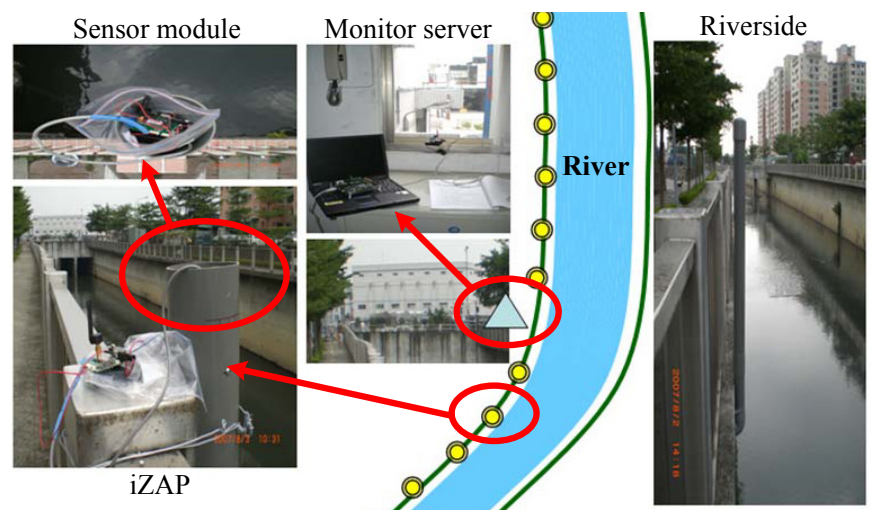

Fig. 4. The field deployment of a LT network.

of the LT WSN is possible due to the existence of neighbor tables and our design of hierarchical network addresses. Therefore, unlike the original ZigBee tree routing, nodes are not restricted to relay packets only to their parents or children.

\section{Performance Evaluations}

\section{A. Prototyping Experience}

We implement a ZigBee-based wireless sensor node, named III ZigBee Advanced Platform (iZAP). The RF module is the Jennic JN5121 with chip antenna [10]. iZAP provides extra I/O pins, which can connect to sensor modules. In the iZAP, we also implement an external watchdog timer (WDT), which can be used to reset the device when an abnormal event occurs. There are three LEDs and an RS232 connector on the device. More details of iZAP can be found in [9].

A LT WSN is deployed to monitor the water level of a river in Taipei County, Taiwan as shown in Fig. 4. There are 41 nodes in the network. The distance between two nodes is 100 meters and the network depth is 20 . There are three clusters in the network and the cluster of the coordinator has only one member (the coordinator itself). Nodes report the sensed readings to the coordinator every minute. The coordinator passes the received sensory readings to a monitor server (as shown in Fig. 5), which can show the statuses of the environment and nodes. The system manager can also use the monitor server to issue commands to the network nodes.

We record the average report latencies of nodes every one hour for two days. Nodes use either the proposed routing protocol or the ZigBee tree routing protocol to report sensory data. When using the ZigBee protocol, we mutually assign addresses to nodes and restrict that each node can only report data to its parent node. Fig. 6 shows the experimental results. We can observe that, in average, our protocol can perform better than ZigBee. This result also indicates that, when using our protocol, the stability of the network can be better.

\section{B. Simulation Results}

We simulate the proposed routing protocol in a large scale LT network, whose layout is the same as the one in Fig. 2(b). The distance between two adjacent nodes is $20 \mathrm{~m}$. The simulation program randomly generates source-destination pairs and 


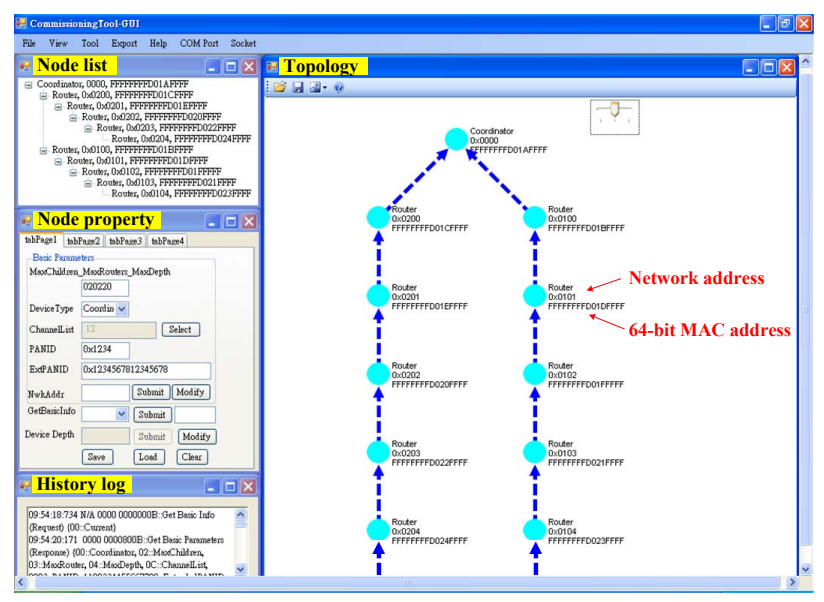

Fig. 5. The graphical user interface of the monitor server.

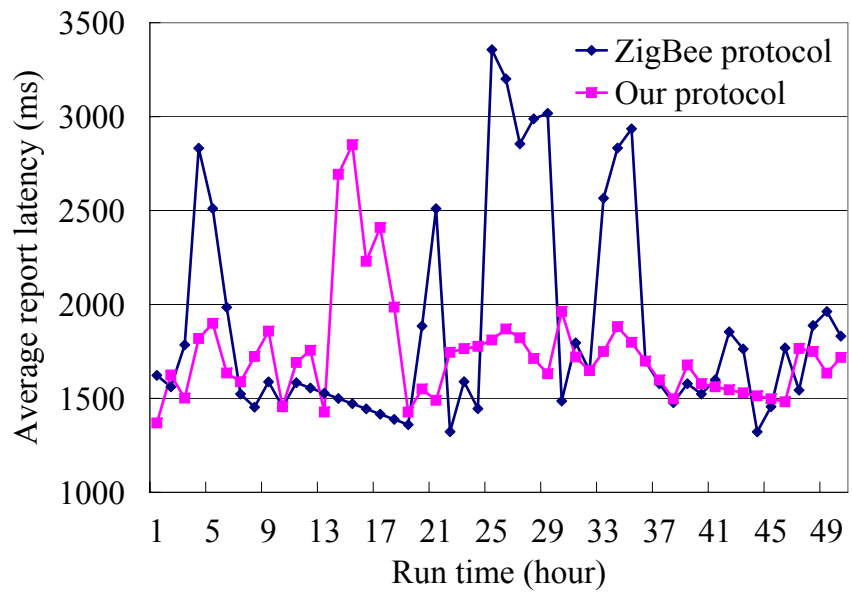

Fig. 6. Comparison on the measured report latency.

records the averaged hop counts from sources to destinations. Fig. 7 shows the simulation results. We can observe that, when transmission ranges become large, the average number of hop count decreases. This result indicates that shortcuts can effectively reduce the number of transmissions.

\section{Conclusions}

We have proposed address assignment and routing schemes for ZigBee-based LT WSNs. The proposed address assignment scheme divides nodes into several clusters and then assigns each node a cluster ID and a node ID as its network address. The routing protocol uses addresses of nodes to find routing paths and allows nodes to utilize shortcuts. We verify both our schemes by real implementation and simulations. In the future, we plan to discuss address assignment and routing schemes for other specific but common topologies such as hypercube networks.

\section{ACKNOWLEDGEMENTS}

Y.-C. Tseng's research is co-sponsored by Taiwan MoE ATU Program, by NSC grants 93-2752-E-007-001-PAE, 962623-7-009-002-ET, 95-2221-E-009-058-MY3， 95-2221-E-

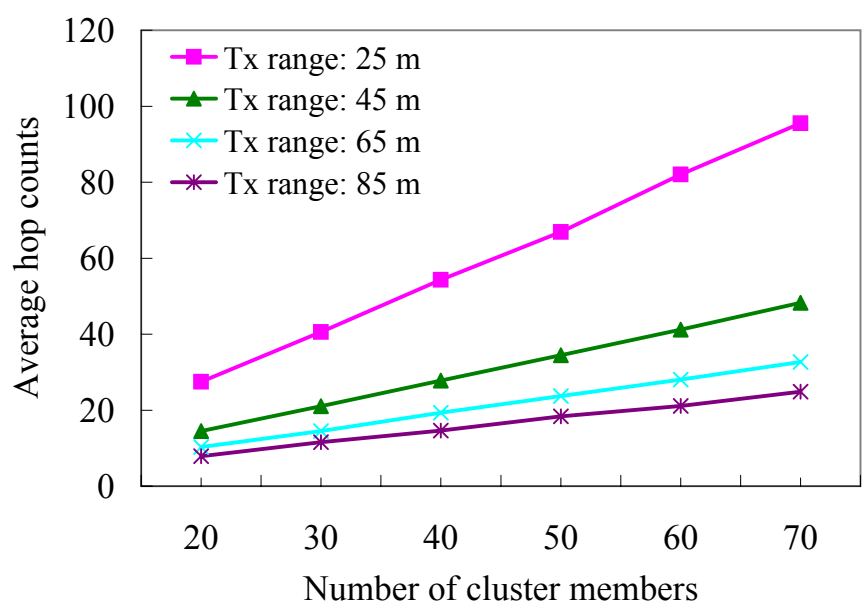

Fig. 7. Simulation on averaged number of hop counts when transmission ranges of nodes and the number of cluster members are varied.

009-060-MY3, 95-2219-E-009-007, 95-2218-E-009-209, and 94-2219-E-007-009, by Realtek Semiconductor Corp., by MOEA under grant number 94-EC-17-A-04-S1-044, by ITRI, Taiwan, by Microsoft Corp., and by Intel Corp.

\section{REFERENCES}

[1] A. A. Ahmed, H. Shi, and Y. Shang. SHARP: A new approach to relative localization in wireless sensor networks. In Proc. of Int'l Conference on Distributed Computing Systems Workshops (ICDCSW), 2005.

[2] M. Ali and Z. A. Uzmi. An energy-efficient node address naming scheme for wireless sensor networks. In Proc. of IEEE Int'l Networking and Communications Conference (INCC), 2004.

[3] D. Braginsky and D. Estrin. Rumor routing algorithm for sensor networks. In Proc. of ACM Int'l Workshop on Wireless Sensor Networks and Applications (WSNA), 2002.

[4] Habitat monitoring on great duck island. http://www.greatduckisland.net/technology.php.

[5] Dust network Inc. http://dust-inc.com/flash-index.shtml.

[6] Design and construction of a wildfire instrumentation system using networked sensors. http://firebug.sourceforge.net/.

[7] C.-F. Huang, Y.-C. Tseng, and L.-C. Lo. The coverage problem in three-dimensional wireless sensor networks. In Proc. of IEEE Global Telecommunications Conference (Globecom), 2004.

[8] IEEE standard for information technology - telecommunications and information exchange between systems - local and metropolitan area networks specific requirements part 15.4: wireless medium access control (MAC) and physical layer (PHY) specifications for low-rate wireless personal area networks (LR-WPANs), 2003.

[9] III ZigBee Advanced Platform (iZAP). http://zigbee.iii.org.tw/.

[10] Jennic JN5121. http://www.jennic.com/.

[11] Motes, smart dust sensors, wireless sensor networks. http://www.xbow.com/.

[12] E. Ould-Ahmed-Vall, D. M. Blough, B. S. Heck, and G. F. Riley. Distributed unique global ID assignment for sensor networks. In Proc. of IEEE Mobile Adhoc and Sensor Systems Conference (MASS), 2005.

[13] C. Schurgers, G. Kulkarni, and M. B. Srivastava. Distributed on-demand address assignment in wireless sensor networks. IEEE Trans. Parallel Distributed System, 13(10):1056-1065, 2002.

[14] Y.-C. Tseng, M.-S. Pan, and Y.-Y. Tsai. Wireless sensor networks for emergency navigation. IEEE Computer, 39(7):55-62, 2006.

[15] W. Ye, J. Heidemann, and D. Estrin. An energy-efficient MAC protocol for wireless sensor networks. In Proc. of IEEE INFOCOM, 2002.

[16] H. Zhou, M. W. Mutka, and L. M. Ni. Reactive ID assignment for sensor networks. In Proc. of IEEE Mobile Adhoc and Sensor Systems Conference (MASS), 2005.

[17] ZigBee-2006 specification, ZigBee document 064112, 2006. 